DOI: $10.18027 / 2224-5057-2021-11-3 s 2-13$

Цитирование: Хохлова С.В., Коломиец Л.А., Кравец О.А., Морхов К.Ю., Нечушкина В.М., Тюляндина А.С. и соавт. Практические рекомендации по лекарственному лечению рака шейки матки. Злокачественные опухоли : Практические рекомендации RUSSCO \#3s2, 2021 (том 11). 13

\title{
ПРАКТИЧЕСКИЕ РЕКОМЕНДАЦИИ ПО ЛЕКАРСТВЕННОМУ ЛЕЧЕНИЮ РАКА ШЕЙКИ МАТКИ
}

Коллектив авторов: Хохлова С.В., Коломиец Л.А., Кравец О.А., Морхов К.Ю., Нечушкина В.М., Тюляндина А.С., Урманчеева А.Ф.

Ключевые слова: рак шейки матки, вирус папилломы человека

Рак шейки матки (РШМ) - одно из наиболее часто встречающихся злокачественных новообразований у женщин в мире. Причиной РШМ является ВПЧ онкогенных подтипов. Онкогенные подтипы ВПЧ 16/18 обнаруживаются у большинства больных РШМ. В настоящее время в развитых странах широко используется тестирование ДНК ВПЧ. Основным методом скрининга РШМ является цитологическое исследование мазков по Папаниколау.

\section{1. КЛАССИФИКАЦИЯ И ОПРЕДЕЛЕНИЕ СТАДИИ}

Диагноз РШМ устанавливается только на основании результатов гистологического исследования.

Для определения стадии РШМ возможно использование любых методов визуализации и/или данных морфологического исследования. В регионах с ограниченными ресурсами возможно установление стадии на основании клинических данных. Стадия РШМ устанавливается до начала специальной терапии, что необходимо для выбора метода и составления плана лечения. Стадия РШМ не изменяется после начала специального лечения. В случаях, когда есть затруднения в точном определении стадии, следует устанавливать более раннюю. Морфологические находки у больных, подвергнутых хирургическому лечению, должны быть отмечены отдельно. Для этой цели подходит номенклатура TNM (буквенной аббревиатурой перед TNM необходимо указывать, на основании каких данных определен статус первичной опухоли (T), лимфатических узлов (N) и отдаленных метастазов (M): клинические данные (сTNM), данные лучевых методов обследования (iTNM), данные послеоперационного морфологического исследования (рTNM).

Определение стадии производится на основании классификации FIGO (2009 г.) и TNM (8-е издание, 2016 г.) 
Таблица 1. Классификация РШМ по стадиям (FIGO, 2009 г.) и распространенности опухолевого процесса (TNM, 2016 г.)

\begin{tabular}{|c|c|c|c|c|}
\hline TNM & \multicolumn{4}{|l|}{ FIGO } \\
\hline TX & \multicolumn{4}{|c|}{ Недостаточно данных для оценки первичной опухоли } \\
\hline TO & \multicolumn{4}{|c|}{ Первичная опухоль не определяется } \\
\hline Tis1 & \multicolumn{4}{|c|}{ Рак in situ, преинвазивный рак (эти случаи не входят в статистику заболеваемости РШМ) шейки матки) } \\
\hline T1 & Стадия $\mathrm{I}^{2}$ & \multicolumn{3}{|c|}{ Опухоль ограничена маткой (распространение на тело матки не учитывается) } \\
\hline T1a & IA & \multicolumn{3}{|c|}{ Диагноз устанавливается только при гистологическом исследовании } \\
\hline T1a1 & IA1 & \multicolumn{3}{|c|}{$\begin{array}{l}\text { Глубина инвазии не более } 3 \text { мм (микроинвазивный рак); горизонтальное рас- } \\
\text { пространение не должно превышать } 7 \text { мм, в противном случае опухоль следует } \\
\text { относить к стадии IB }\end{array}$} \\
\hline T1a2 & IA2 & \multicolumn{3}{|c|}{$\begin{array}{l}\text { Глубина инвазии более } 3 \text { мм, но не более } 5 \text { мм (измеряется от базальной мембраны } \\
\text { поверхностного или железистого эпителия); горизонтальное распространение } \\
\text { не должно превышать } 7 \text { мм, в противном случае опухоль следует относить к стадии } \\
\text { IB }\end{array}$} \\
\hline T1b & IB & \multicolumn{3}{|l|}{ Глубина инвазии более 5 мм } \\
\hline T1b1 & IB1 & \multicolumn{3}{|c|}{ Размеры опухоли не более 4 см } \\
\hline $\mathrm{T}_{1} \mathrm{~b}^{2}$ & IB2 & \multicolumn{3}{|l|}{ Размеры опухоли более 4 см } \\
\hline $\mathrm{T} 2$ & Стадия $\|^{3}$ & \multicolumn{3}{|c|}{$\begin{array}{l}\text { Опухоль, распространившаяся за пределы матки, без перехода на стенки таза } \\
\text { и поражения нижней трети влагалища }\end{array}$} \\
\hline T2a & IIA & \multicolumn{3}{|l|}{ Без вовлечения параметрия } \\
\hline T2a1 & IIA1 & \multicolumn{3}{|c|}{ Размеры опухоли не более 4 см } \\
\hline $\mathrm{T} 2 \mathrm{a} 2$ & IIA2 & \multicolumn{3}{|l|}{ Размеры опухоли более 4 см } \\
\hline $\mathrm{T} 2 \mathrm{~b}$ & IIB & \multicolumn{3}{|l|}{ С вовлечением параметрия } \\
\hline T3 & Стадия III ${ }^{4}$ & \multicolumn{3}{|c|}{$\begin{array}{l}\text { Опухоль, вовлекающая стенки таза (при ректальном исследовании отсутствует } \\
\text { пространство между опухолью и стенкой таза) или нижнюю треть влагалища, } \\
\text { а также все случаи РШМ с гидронефрозом и нефункционирующей почкой }\end{array}$} \\
\hline ТЗа & IIIA & \multicolumn{3}{|c|}{ Вовлечение нижней трети влагалища } \\
\hline T3b & IIIB & \multicolumn{3}{|c|}{ Распространение на стенку таза или гидронефроз и нефункционирующая почка } \\
\hline & Стадия IV & \multicolumn{3}{|c|}{$\begin{array}{l}\text { Опухоль, распространившаяся за пределы таза или прорастающая слизистую } \\
\text { мочевого пузыря или прямой кишки (буллезный отек не позволяет отнести опухоль } \\
\text { к стадии IV) }\end{array}$} \\
\hline T4 & IVA $^{5}$ & \multicolumn{3}{|c|}{ Прорастание мочевого пузыря или прямой кишки } \\
\hline M1 & IVB & \multicolumn{3}{|l|}{ Отдаленные метастазы } \\
\hline \multicolumn{3}{|c|}{ Состояние регионарных лимфатических узлов } & \multicolumn{2}{|c|}{ Отдаленные метастазы } \\
\hline NX & \multicolumn{2}{|c|}{ Недостаточно данных для оценки } & MO & $\begin{array}{l}\text { Отдаленные метастазы } \\
\text { отсутствуют }\end{array}$ \\
\hline NO & \multicolumn{2}{|c|}{$\begin{array}{l}\text { Признаков поражения регионарных } \\
\text { лимфатических узлов нет }\end{array}$} & $M 1^{6}$ & Имеются отдаленные метастазы \\
\hline N1 & \multicolumn{4}{|c|}{ Метастазы в регионарных лимфатических узлах } \\
\hline
\end{tabular}


1 В классификации FIGO стадию 0 (Tis) не применяют.

2 Cтадии IA1 и IA2 диагностируют на основании результатов гистологического исследования операционных препаратов преимущественно после конизации шейки матки. При конизации должна быть удалена вся измененная влагалищная часть шейки матки. При макроскопически определяемой опухоли ее следует относить к стадии T1b. Глубина инвазии определяется от базальной мембраны, независимо от того, исходит опухоль из поверхностного эпителия или из железистого. Выявление опухолевых эмболов в сосудахне влияет на стадию, но должно быть отражено в заключении гистологического исследования, поскольку может повлиять на тактику лечения. Распространение на тело матки не учитывается, поскольку переход опухоли на тело матки до операции выявить очень трудно.

з При вовлечении параметрия стадия III диагностируется тогда, когда инфильтрат в параметрии имеет узловую форму и доходит до стенки таза. В остальных случаях диагностируется стадия IIb. Следует подчеркнуть, что при гинекологическом исследовании судить о природе инфильтрата (воспалительный или опухолевый) невозможно.

${ }_{4}$ Пригидронефрозе и нефункционирующей почке, обусловленныхсдавлением мочеточника опухолью, диагностируют стадию III.

5 Инвазия в слизистую оболочку мочевого пузыря или прямой кишки должна быть подтверждена результатами биопсии.

6 Включая метастазы в паховых, поясничных лимфатическихузлах, а также на брюшине, за исключением серозных оболочек органов малого таза. Исключены метастазы во влагалище, серозных оболочках малого таза и придатках.

В 2018 г. опубликована новая классификация FIGO (табл. 2). Однако до настоящего времени нет пересмотра классификации TNM. В России определение стадии РШМ по-прежнему производится на основании классификации FIGO (2009 г.) и TNM (8-е издание, 2016 г.).

Таблица 2. Классификация РШМ по стадиям (FIGO, 2018 г.)

\begin{tabular}{|c|c|}
\hline Стадия I & Опухоль ограничена шейкой матки (распространение на тело матки не учитывается) \\
\hline IA & $\begin{array}{l}\text { Инвазивная карцинома, диагностирующаяся только микроскопически, с наибольшей } \\
\text { глубиной инвазии } \leq 5 \text { мм }^{1,2}\end{array}$ \\
\hline IA1 & Стромальная инвазия< 3 мм \\
\hline IA2 & Стромальная инвазия $\geq 3$ и < 5 мм \\
\hline IB & $\begin{array}{l}\text { Инвазивная карцинома с глубиной инвазии } \geq 5 \text { мм (больше, чем стадия IA), опухоль ограничена } \\
\text { шейкой матки }\end{array}$ \\
\hline IB1 & $\begin{array}{l}\text { Инвазивная карцинома с глубиной инвазии } 25 \text { мм и размером опухоли< } 2 \text { см в наибольшем } \\
\text { измерении }\end{array}$ \\
\hline IB2 & Инвазивная карцинома с размером опухоли 22 и < 4 см в наибольшем измерении \\
\hline IB3 & Инвазивная карцинома с размером опухоли 4 см в наибольшем измерении \\
\hline Стадия II & $\begin{array}{l}\text { Опухоль, распространившаяся за пределы матки, без перехода на стенки таза и поражения } \\
\text { нижней трети влагалища }\end{array}$ \\
\hline IIA & Опухоль поражает до двух третей верхней части влагалища без вовлечения параметрия \\
\hline IIA1 & Инвазивная карцинома с размером опухоли< 4 см в наибольшем измерении \\
\hline IIA2 & Инвазивная карцинома с размером опухоли 4 см в наибольшем измерении \\
\hline IIB & С вовлечением параметрия, но без распространения на стенку таза \\
\hline
\end{tabular}




\begin{tabular}{|l|l|}
\hline Стадия III & $\begin{array}{l}\text { Опухоль распространяется на стенку малого таза и/или вовлекает нижнюю треть влагалища } \\
\text { и/или является причиной гидронефроза или нефункционирующей почки и/или поражение } \\
\text { тазовых и/или парааортальных лимфатических узлов }\end{array}$ \\
\hline IIIA & Опухоль вовлекает нижнюю треть влагалища, но не распространяется на стенку таза \\
\hline IIIB & $\begin{array}{l}\text { Опухоль распространяется на стенку таза и/или является причиной гидронефроза } \\
\text { или нефункционируюей почки }\end{array}$ \\
\hline IIIC & $\begin{array}{l}\text { Поражение тазовых и/или парааортальных лимфатических узлов независимо от размера } \\
\text { и распространения первичной опухоли (с обозначением индексов г и р) }\end{array}$ \\
\hline IIIC1 & Поражение только тазовых лимфатических узлов \\
\hline IIIC2 & Поражение парааортальных лимфатических узлов \\
\hline Cтадия IV & $\begin{array}{l}\text { Опухоль, распространившаяся за пределы таза или прорастающая слизистую мочевого } \\
\text { пузыря или прямой кишки (буллезный отек не позволяет отнести опухоль к стадии IV) }\end{array}$ \\
\hline IVA & Прорастание мочевого пузыря или прямой кишки ${ }^{4}$ \\
\hline IVB & Отдаленные метастазы \\
\hline
\end{tabular}

1 Методы визуализации и гистологическое исследование могут быть использованы на всех этапах в дополнение кклиническим данным о размере опухоли и ее распространении.

2 При стадии ІА горизонтальное распространение опухоли больше не учитывается. Выявление опухолевых эмболов в сосудах не меняет стадию, но должно быть отражено в заключении гистологического исследования, посколькуможет повлиять на тактикулечения

3 Добавление обозначений индексов r (методы визуализации) и р (гистологическое исследование) необходимо для уточнения метода, на основании которого установлена стадия IIIC. Например, если метастатическое поражение тазовых лимфатических узлов выявлено методами визуализации, стадия будет IIIC1r, nри гистологическом подтверждении поражения тазовых лимфатических узлов - IIIC1р. При этом метод визуализации или методика гистологического исследования должны быть описаны в заключении. При затруднениях в точном определении стадии следует устанавливать более раннюю.

4 Инвазия в слизистую оболочку мочевого пузыря или прямой кишки должна быть подтверждена результатами биопсии.

5 Включая метастазы в паховых лимфатических узлах, а также на брюшине, за исключением серозных оболочек органов малого таза. Исключены метастазы во влагалище, серозных оболочках органов малого таза и придатках.

Регионарными лимфатическими узлами при РШМ считаются наружные, внутренние, общие подвздошные, параметральные, запирательные и крестцовые парааортальныем (FIGO, 2018 г.).

У 70-80\% больных инвазивным РШМ диагностируется плоскоклеточный рак, у 10-20\% аденокарцинома и у $10 \%$ - низкодифференцированный рак. Другие гистологические типы злокачественных опухолей шейки матки составляют не более $1 \%$. 
Таблица 3. Морфологическая классификация злокачественных опухолей шейки матки (ВO3, 2020 г.)

ОПУХОЛИ ИЗ ПЛОСКОГО ЭПИТЕЛИЯ

- Плоскоклеточные интраэпителиальные поражения (поражение плоского эпителия (SIL)):

- поражение плоского эпителия легкой степени (LSIL)

- поражение плоского эпителия тяжелой степени (HSIL)

- Плоскоклеточный рак HPV-ассоциированный

- Плоскоклеточный рак HPV-не ассоциированный

- Плоскоклеточный рак неспецифического типа

ОПУХОЛИ ИЗ ЖЕЛЕЗИСТОГО ЭПИТЕЛИЯ

- Аденокарцинома in situ

- Аденокарцинома in situ HPV-ассоциированная

- Аденокарцинома in situ HPV-не ассоциированная

- Аденокарцинома неспецифического типа

- Аденокарцинома HPV-ассоциированная

- АденокарциномаHPV-не ассоциированная желудочного типа

- АденокарциномаHPV-не ассоциированная светлоклеточного типа

- АденокарциномаHPV-не ассоциированная мезонефроидного типа

- АденокарциномаHPV-не ассоциированная неспецифического типа

- Эндометриоидная аденокарцинома неспецифического типа

- Карциносаркома нескпецифического типа

- Аденоплоскоклеточная карцинома

- Мукоэпидермоидная карцинома

- Аденоидно-базальная карцинома

- Карцинома недифференцированная неспецифического типа

СМЕШАННЫЕ ЭПИТЕЛИАЛЬНЫЕ И МЕЗЕНХИМАЛЬНЫЕ ОПУХОЛИ

- Аденосаркома

ГЕРМИНОГЕННЫЕ ОПУХОЛИ

- Герминогенные опухоли неспецифического типа

- зрелая тератома неспецифического типа

- опухоль желточного мешка неспецифического типа

- опухоль эндодермального синуса

- хориокарцинома

- дермоидная киста неспецифического типа

НЕЙРОЭНДОКРИННЫЕ ОПУХОЛИ

- Нейроэндокринные карциномы

- мелкоклеточная нейроэндокринная карцинома

- крупноклеточная нейроэндокринная карцинома

- Смешанные нейроэндокринные-ненейроэндокринные опухоли

- Аденокарцинома смешанная с нейроэндокринной карциномой

\section{2. ДИАГНОСТИКА}

- Физикальное исследование, включающее гинекологический осмотр и ректовагинальное исследование

- Кольпоскопия, биопсия всех подозрительных участков шейки матки, при необходимости - конизация 
- Рекомендуется проводить гистологическое исследование хирургически удаленного опухолевого препарата, при этом в морфологическом заключении рекомендуется отразить следующие параметры:

1) гистологический тип опухоли;

2) степень дифференцировки опухоли;

3) размеры опухоли (три размера);

4) глубину инвазии опухоли;

5) толщину шейки матки в месте наибольшей инвазии опухоли;

6) наличие/отсутствие опухолевхе эмболов в кровеносныхи лимфатических сосудах;

7) опухоль в крае резекции влагалища, параметриев, по границам резекции шейки матки (при выполнении конизации/ампутации или трахелэктомии); при близком крае резекции указывается расстояние от края резекции до опухоли;

8) переход опухоли на влагалище;

9) опухолевое поражение параметрия справа, слева;

10) общее число удаленных и поражённых лимфоузлов параметрия справа, слева; размер метастазов в мм (изолированные опухолевые клетки, микрометастазы, макрометастазы);

11) общее число удаленных и поражённых тазовых лимфоузлов справа, слева; размер метастазов в мм (изолированные опухолевые клетки, микрометастазы, макрометастазы);

12) общее число удаленных и поражённых поясничных лимфоузлов; размер метастазов в мм (изолированные опухолевые клетки, микрометастазы, макрометастазы);

13) переход опухоли на тело матки;

14) метастатическое поражение яичников;

15) прорастание опухоли в стенку мочевого пузыря, прямой кишки (при выполнении экзентерации или резекции смежных органов);

16) степень лечебного патоморфоза первичной опухоли и метастазов в лимфоузлах в случае предоперационной терапии со ссылкой на классификацию, по которой оценивается степень лечебного патоморфоза.

- Клинический анализ крови, биохимический анализ крови (показатели функции печени и почек), коагулограмма, общий анализ мочи, ЭКГ

- $\quad$ R-графия органов грудной клетки

- Ц Цистоскопия (по показаниям)

- Ректороманоскопия (по показаниям)

- Экскреторная урография, ренография (по показаниям)

- УзИ органов брюшной полости, малого таза, забрюшинного пространства, паховых лимфоузлов

- УзИ шейно-надключичных лимфоузлов (при наличии метастазов в забрюшинных лимфоузлах)

- Определение уровня SCC в сыворотке (при плоскоклеточном раке)

- МРТ органов малого таза с в/в контрастированием (МРТ информативнее КТ при оценке глубины инвазии и перехода опухоли на параметрий и смежные органы) 
- КТ малого таза и забрюшинного пространства с в/в контрастированием (по показаниям) (при выявлении метастазов в лимфатических узлахинформативность КТ и МРТ одинакова)

- ПЭТ/КТ (по показаниям) (наиболее информативна для выявления поражения лимфатических узлов и отдаленных метастазов)

- Колоноскопия (по показаниям)

- Рентгенография или сцинтиграфия костей скелета (при подозрении на метастатическое поражение костей)

- МРТ/КТ головного мозга с в/в контрастированием (при подозрении на метастатическое поражение головного мозга)

- К КТМРТ органов брюшной полости (по показаниям)

- КТ/МРТ органов грудной клетки (по показаниям).

\section{3. ЛЕЧЕНИЕ}

В табл. 4 представлены две классификации типов хирургических вмешательств при РШМ по Piver M.S., Rutledge F. (1974 г.) и Querleu D., Morrow C.P. (2008 г.).

Таблица 4. Классификация типов хирургических вмешательств при РШМ

\begin{tabular}{|c|c|c|c|}
\hline \multicolumn{4}{|c|}{ КлассификацияPiverM. S, Rutledge F. (1974 г.) } \\
\hline І тип & \multicolumn{3}{|l|}{ Экстрафасциальная экстирпация матки } \\
\hline II тип & \multicolumn{3}{|c|}{$\begin{array}{l}\text { Модифицированная расширенная экстирпация матки подразумевает удаление медиальной } \\
\text { трети кардинальных, пузырно-маточных и крестцово-маточных связок, верхней трети влагалища } \\
\text { и тазовую лимфаденэктомию. Мочеточники туннелируются, но не мобилизуются по нижне- } \\
\text { латеральной полуокружности. Маточные сосуды пересекаются на уровне мочеточника }\end{array}$} \\
\hline III тип & \multicolumn{3}{|c|}{$\begin{array}{l}\text { Расширенная экстирпация матки по Мейгсу подразумевает полное удаление кардинальных } \\
\text { и крестцово-маточных связок от стенок таза, пузырно-маточной связки-от стенки мочевого } \\
\text { пузыря, верхней трети влагалища и тазовую лимфаденэктомию. Мочеточники мобилизуются } \\
\text { полностью до места впадения в мочевой пузырь. Маточные сосуды пересекаются у места } \\
\text { отхождения от внутренних подвздошных сосудов }\end{array}$} \\
\hline IV тип & \multicolumn{3}{|c|}{$\begin{array}{l}\text { Расширенная экстирпация матки включает пересечение верхней пузырной артерии, удаление } \\
\text { периуретеральной клетчатки и до 3/4 влагалища }\end{array}$} \\
\hline V тип & \multicolumn{3}{|c|}{$\begin{array}{l}\text { Комбинированная расширенная экстирпация матки подразумевает удаление дистальных отделов } \\
\text { мочеточников и резекцию мочевого пузыря }\end{array}$} \\
\hline \multicolumn{4}{|c|}{ Классификация Querleu D., Morrow C. P. (2017 г.) } \\
\hline Тип & Латеральный параметрий & Передний параметрий & Задний параметрий \\
\hline A & $\begin{array}{l}\text { Середина расстояния между шейкой матки } \\
\text { и мочеточником (мочеточник не мобилизуется) }\end{array}$ & Минимально & Минимально \\
\hline B1 & $\begin{array}{l}\text { На уровне мочеточника (мочеточник } \\
\text { мобилизуется от латерального параметрия) }\end{array}$ & Частичное удаление & Частичное удаление \\
\hline B2 & В1+ парацервикальные лимфоузлы & B1 & B1 \\
\hline
\end{tabular}




\begin{tabular}{|c|c|c|c|}
\hline C1 & $\begin{array}{l}\text { Верхняя часть до стенки таза (латерально) и до } \\
\text { уровня глубокой маточной вены (вертикально) }\end{array}$ & $\begin{array}{l}\text { Верхняя часть (над } \\
\text { мочеточником) } \\
\text { на уровне мочевого } \\
\text { пузыря }\end{array}$ & $\begin{array}{l}\text { Верхняя часть } \\
\text { до уровня } \\
\text { прямой кишки (гипо- } \\
\text { гастральные нервы } \\
\text { сохраняются) }\end{array}$ \\
\hline C2 & Полностью до стенки таза & $\begin{array}{l}\text { Полностью } \\
\text { до мочевого пузыря }\end{array}$ & $\begin{array}{l}\text { Полностью } \\
\text { до крестца }\end{array}$ \\
\hline D1 & $\begin{array}{l}\text { Удаление внутренних подвздошных сосудов } \\
\text { и клетчатки за ними }\end{array}$ & $\mathrm{C} 2$ & $\mathrm{C} 2$ \\
\hline D2 & $\begin{array}{l}\text { Резекция примыкающих фасциальных } \\
\text { и мышечных структур стенки таза }\end{array}$ & $\mathrm{C} 2$ & $\mathrm{C} 2$ \\
\hline
\end{tabular}

Выбор метода лечения РШМ определяется индивидуально и зависит от распространенности опухолевого процесса и тяжести сопутствующей соматической патологии.

\section{1. ІА1стадия (инвазия $\leq 3$ мм; горизонтальное распространение $\leq 7$ мм)}

Адекватным объемом хирургического вмешательства при РШМ IA1 стадии в отсутствие эмболов в лимфатических щелях является конизация шейки матки с выскабливанием оставшейся части цервикального канала и, по показаниям, полости матки. При отсутствии опухоли в крае резекции и соскобе из оставшейся части цервикального канала выполнение экстирпации матки нецелесообразно.

При обнаружении в краях резекции шейки матки или в соскобе из оставшейся части цервикального канала дисплазии (CINIII) или клеток рака необходимо провести повторную конизацию. При невозможности реконизации проводится лечение как при IB1 стадии.

При наличии лимфоваскулярной инвазии и отсутствии необходимости сохранения детородной функции показана экстирпация матки (операция I типа) или модифицированная экстирпация матки (операция II типа) и тазовая лимфодиссекция (возможна биопсия сторожевого лимфоузла). У молодых пациенток (до 45 лет) при удалении матки возможно сохранить функцию яичников, выполнив ихтранспозицию.

При наличии лимфоваскулярной инвазии и необходимости сохранения детородной функции показана конизация шейки матки с выскабливаем оставшейся части цервикального канала или трахелэктомия (операция I или II типа) и тазовая лимфодиссекция (возможна биопсия сторожевого лимфоузла).

\section{2. ІА2 стадия (инвазия $\geq 3$ мм и $\leq 5$ мм)}

Стандартным хирургическим доступом является лапаротомия. Проспективное рандомизированное исследование продемонстрировало статистически значимо меньшую общую и безрецидивную выживаемость у больных РШМ IA2-IB1 стадий, оперированных в объеме расширенной экстирпации матки лапароскопическим доступом по сравнению с открытым доступом. В связи с этим пациентки РШМ IA2-IB1 стадий должны быть ознакомлены с результатами данного исследования и предупреждены о рисках ухудшения отдаленных 
онкологических результатов лечения при выполнении расширенной экстирпации матки лапароскопическим доступом.

Показано выполнение модифицированной расширенной экстирпации матки (тип II/B) и тазовой лимфодиссекции (возможна биопсия сторожевого лимфоузла). У молодых пациенток (до 45 лет) возможно сохранить функцию яичников, выполнив их транспозицию.

Молодым пациенткам, желающим сохранить фертильность, независимо от лимфоваскулярной инвазии и при отсутствии метастазов в тазовых лимфатических узлах, может быть предложена расширенная трахелэктомия (тип II/B).

При отсутствии лимфоваскулярной инвазии у молодых больных, желающих сохранить фертильность, возможно выполнение ультраконсервативного лечения в объеме ампутации шейки матки, выскабливания оставшейся части цервикального канала (отсутствие опухоли в крае резекции и соскобе из оставшейся части цервикального канала) и тазовой лимфодиссекции (возможна биопсия сторожевого лимфоузла).

При противопоказаниях к хирургическому лечению или по желанию больной может проводиться лт.

\subsection{IB1 и IIA1 стадии (опухоль $\leq 4$ см)}

Применение комбинации хирургического лечения и лТ статистически значимо увеличивает частоту осложнений. Поэтому при планировании лечения больных С IB1 и IIA1 стадиями РШМ следует избегать комбинации хирургического лечения и лТ.

При IB1 (опухоль $\leq 4$ см) стандартным хирургическим доступом является лапаротомия. Проспективное рандомизированное исследование продемонстрировало статистически значимо меньшую общую и безрецидивную выживаемость у больных РШМ IA2-IB1 стадий, оперированных в объеме расширенной экстирпации матки лапароскопическим доступом по сравнению с открытым доступом. В связи с этим пациентки РШМ IA2-IB1 стадий должны быть ознакомлены с результатами данного исследования и предупреждены о рисках ухудшения отдаленных онкологических результатов лечения при выполнении расширенной экстирпации матки лапароскопическим доступом.

При IB1 и IIA1 стадиях (опухоль $\leq 2$ см) рекомендована модифицированная расширенная экстирпация матки (тип II/В или С1). У молодых пациенток (до 45 лет) при удалении матки возможно сохранить функцию яичников, выполнив их транспозицию.

Согласно данным проспективных и ретроспективных исследований, больным РШМ IB1 стадии (опухоль $\leq 2$ см) при глубине инвазии менее 1 см без лимфоваскулярной инвазии и метастазов в тазовых лимфатических узлах возможно выполнение ультраконсервативного лечения в объеме экстирпации матки (тип I) и тазовой лимфодиссекции. У молодых пациенток (до 45 лет) при удалении матки возможно сохранить функцию яичников, выполнив их транспозицию.

При IB1 и IIA1 стадиях (опухоль >2 см) рекомендована расширенная экстирпация матки (тип III/C2). У молодых пациенток (до 45 лет) при удалении матки возможно сохранить функцию яичников, выполнив их транспозицию.

При IB1 и IIA1 стадий (опухоль < 4 см) при наличии метастазов в тазовых лимфатических узлах рекомендовано выполнение парааортальной лимфодиссекции. При выполнении 
парааортальной лимфодиссекции показано удаление паракавальных, прекавальных, аортокавальных, преаортальных, парааортальных лимфатических узлов как минимум до уровня нижней брыжеечной артерии. По показаниям (данные ревизии или лучевых методов диагностики) возможно выполнение поясничной лимфодиссекции до уровня почечных сосудов. При этом удаление матки с первичной опухолью не обязательно (прерванная гистерэктомия) ввиду необходимости проведения послеоперационной ХлТ.

По данным проспективных исследований при наличии метастазов в тазовых лимфатических узлах с одной стороны и отсутствии метастатического поражения поясничных лимфатических узлов возможно выполнение расширенной параметрэктомии (тип D1) на стороне поражения. Выполнение данного вмешательства возможно только группой квалифицированных специалистов, имеющих соответствующий опыт. При выполнении расширенной параметрэктомии адъювантная лТ не показана. Возможно проведение адъювантной ХТ (табл. 6, «Химиотерапия І линии»).

При невозможности хирургического лечения по медицинским показаниям или в связи с отказом пациентки рекомендуется ЛТ или ХЛТ по радикальной программе. Адъювантная гистерэктомия после ЛТ или ХЛТ по радикальной программе не рекомендуется.

Адъювантное лечение проводится в зависимости от наличия факторов риска прогрессирования.

Таблица 5. Факторы риска прогрессирования при раке шейки матки

\begin{tabular}{|l|l|l|}
\hline Высокий риск & Промежуточный риск & Низкий риск \\
\hline $\begin{array}{l}\text { Присутсвует хотя бы 1 из } \\
\text { факторов }\end{array}$ & $\begin{array}{l}\text { Присутствуют хотя бы 2 из } \\
\text { факторов }\end{array}$ & $\begin{array}{l}\text { Отсутствуют факторы либо } \\
\text { присутствует только 1 из факторов }\end{array}$ \\
\hline Инвазия опухоли в параметрий & Лимфоваскулярная инвазия & Лимфоваскулярная инвазия \\
\hline $\begin{array}{l}\text { Метастазы в подвздошных } \\
\text { лимфоузлах }\end{array}$ & $\begin{array}{l}\text { Инвазия опухоли более чем } \\
\text { на 1/3 толщины миометрия шейки } \\
\text { матки }\end{array}$ & $\begin{array}{l}\text { Инвазия опухоли более чем } \\
\text { нат толщины миометрия шейки } \\
\text { матки }\end{array}$ \\
\hline $\begin{array}{l}\text { Опухоль в крае резекции } \\
\text { влагалища }\end{array}$ & Размер первичной опухоли $\geq 4$ см & Размер первичной опухоли $\geq 4$ см \\
\hline
\end{tabular}

Группа высокого риска прогрессирования: показана адъювантная ХЛТ (дистанционная ЛТ +еженедельное введение цисплатина 40 мг/м² на протяжении лт). При наличии опухоли в краях резекции влагалища показана адъювантная ХЛТ (сочетанная ЛТ +еженедельное введение цисплатина 40 мг/м² на протяжении дистанционной ЛТ). При метастазах в поясничных лимфоузлах проводится облучение расширенным полем. Согласно данным проспективных рандомизированных исследований, при выполнении расширенной экстирпации матки (тип III/C2) в случае обнаружения метастазов в тазовых лимфатических узлах или поражения параметриев в качестве адъювантного лечения возможно проведение 6 курсов адъювантной ХТ (табл. 6, «Химиотерапия I линии»). Эта методика лечения возможна только при наличии команды квалифицированных специалистов, имеющих соответствующий опыт. 
Группа промежуточного риска прогрессирования: в случае расширенной экстирпации матки (тип II/В или C1) показана адъювантная дистанционная ЛТ. После расширенной экстирпации матки (тип III/C2) по факторам промежуточного риска адъювантная дистанционная ЛТ не показана (только если выполнен адекватный объем хирургического вмешательства командой квалифицированных специалистов, имеющих соответствующий опыт).

Группа низкого риска прогрессирования: в случае расширенной экстирпации матки (типа II/В или (1) адъювантное лечение не показано.

Согласно данным проспективных рандомизированных исследований, при IB1 и IIA1 стадиях (опухоль < 4 см) после выполнения расширенной экстирпации матки (тип III/C2) в случае выявления метастазов в тазовых лимфатических узлах или поражения параметриев по данным планового гистологического исследования в качестве адъювантного лечения возможно проведение адъювантной ХТ (табл. 6, «Химиотерапия I линии»). Эта методика лечения возможна только при наличии команды квалифицированных специалистов, имеющих соответствующий опыт.

При необходимости сохранить детородную функцию у больных РШМ IB1 стадии (опухоль <2 см) возможно выполнить расширенную трахелэктомию (тип II/В или С1). При необходимости сохранить детородную функцию у больных РШМ IB1 стадии (опухоль $\geq 2$ см) рекомендуется выполнять расширенную трахелэктомию (тип III/C2).

\subsection{IB2 и IIA2 стадии (опухоль > 4 см)}

Комбинация хирургического лечения с лТ крайне нежелательна, так как существенно увеличивает частоту осложнений. Поэтому при планировании лечения больных IB2 и IIA2 стадий РШМ следует избегать комбинации хирургического лечения и ЛТ. Возможны несколько вариантов лечения.

- Стандартом лечения больных IB2 и ІІА2 стадий РШМ является ХЛТ по радикальной программе: сочетанная ЛТ +еженедельное введение цисплатинав дозе 40 мг/м² (5-6 введений) на протяжении ЛТ. Проведение адъювантной гистерэктомии после ХЛТ не рекомендовано. Проведение неоадъювантной ХТ перед проведением лТ или ХЛТ не рекомендовано. Согласно данным рандомизированных исследований, ХЛТ и неоадъювантная ХТ с последующей операцией являются равно эффективными методиками лечения.

- Вторым возможным вариантом лечения больных IB2 и ІІА2 стадий РШМ является неоадъювантная ХТ на основе препаратов платины (табл. 6, «Химиотерапия I линии») с последующей расширенной экстирпацией матки (тип III/C2). Эта методика лечения позволяет снизить вероятность проведения адъювантной лТ или ХЛТ после хирургического лечения.

- Третьим возможным вариантом лечения больных IB2 и IIA2 стадий РШМ является расширенная экстирпация матки (тип III/C2).

Хирургическое лечение больных IB2 и IIA2 стадий РШМ проводится по тем же принципам, что и при IB1 и ІІА1 стадиях РШМ. 
- У больных РШМ IB2 и ІІА2 стадий стандартным хирургическим доступом является лапаротомия. С учетом данных проспективного рандомизированного исследования при РШМ IB1 стадии лапароскопический доступ не рекомендуется.

- У молодых пациенток (до 45 лет) возможно сохранить функцию яичников, выполнив их транспозицию.

- При наличии метастазов в тазовых лимфатических узлах обязательно выполняется поясничная лимфодиссекция. При этом удаление матки с первичной опухолью не обязательно (прерванная гистерэктомия) ввиду необходимости послеоперационной ХлТ. Однако следует помнить, что при размере первичной опухоли более 4 см выше риск ее неизлеченности при проведении ХлТ по сравнению с опухолями менее 4 см.

- Больным с метастазами в поясничных лимфатических узлах адъювантная лТ проводится расширенным полем.

- При наличии метастазов в тазовых лимфатических узлах с одной стороны и отсутствии метастатического поражения поясничных лимфатических узлов возможно выполнение расширенной параметрэктоми (тип D1) на стороне поражения только группой квалифицированных специалистов, имеющих соответствующий опыт. После выполнения расширенной параметрэктомии (тип D1) адъювантная лТ не показана. Возможно проведение адъювантной ХТ (табл. 6, «Химиотерапия I линии»).

- Адъювантное лечение проводится по тем же принципам, что и при IB1 и IIA1 стадиях РШМ (см. раздел 3.3).

- Согласно даннымпроспективных рандомизированныхисследований, после выполнения расширенной экстирпации матки (тип III/C2) в случае выявления метастазов в тазовых лимфатическихузлах или поражения параметриев по данным планового гистологического исследования в качестве адъювантного лечения возможно проведение адъювантной XТ (табл. 6, «Химиотерапия I линии»). Эта методика лечения возможна только при наличии команды квалифицированных специалистов, имеющих соответствующий опыт.

Согласно данным проспективных исследований, при необходимости сохранить детородную функцию у больных РШМ ІВ2 стадии (опухоль > 4 см) ряд авторов допускает возможность выполнения расширенной трахелэктомии (тип III/C2). Изучается применение неоадъювантной ХT перед органосохраняющим лечением у больных РШМ IB2 стадии (опухоль > 4 см).

\subsection{IIB-IVA стадии}

Рекомендованным стандартом является проведение ХЛТ по радикальной программе (сочетанная ЛТ +еженедельное введение цисплатина $40 \mathrm{mг} / \mathrm{M}^{2}$ на протяжении дистанционной ЛТ). лТ по радикальной программе должна проводиться не более 7-8 недель. Увеличение продолжительности ЛТ терапии снижает выживаемость больных.

Больным с IIB-IVA стадиями РШМ не показано проведение неоадъювантнойXТ передХЛТ.

Ряд авторов при РШМ IIB стадии допускают возможность хирургического вмешательства в объеме расширенной экстирпации матки (тип III/C2) на первом этапе или после неоадъювантной ХT (2-3 курса). Это позволяет избежать проведения адъювантной лТ у пациенток без факторов высокого риска прогрессирования (см. раздел 3.3) по данным 
послеоперационного гистологического исследования. У молодых пациенток (до 45 лет) при стадии $\leq$ IIB возможно сохранение функции яичников путем их транспозиции из зоны последующего облучения. Недостатками выполнения операции на первом этапе является риск осложнений, связанных с комбинированным или комплексным лечением.

При метастатическом поражении тазовых и/или поясничных лимфатических узлов возможно выполнение лимфаденэктомии с последующей ХЛТ по радикальной программе. При поражении поясничных лимфатических узлов облучение проводится расширенным полем.

Целесообразность адъювантной ХТ после ХЛТ по радикальной программе сомнительна. Только одно рандомизированное исследование указывает на значительное преимущество использования адъювантной ХТ после ХлТ по радикальной программе. Остальные рандомизированные исследования не показали преимуществ адъювантной XT.

У части больных без перехода опухоли на стенку таза альтернативой Хлт может служить экзентерация малого таза (особенно при наличии свищей). Выполнение этого вмешательства возможно первичным больным IVA стадии РШМ и больным с центральным рецидивом, вовлекающим мочевой пузырь и/или прямую кишку, не переходящим на стенку таза, при отсутствии диссеминации по брюшине, регионарных и отдаленных метастазов. Чаще выполняется тотальная экзентерация малого таза (включающая удаление мочевого пузыря, матки и прямой кишки).

\subsection{IVB стадия}

Рекомендуется системная ХT (см. раздел 3.8.). Вопрос о ЛТ отдельных метастатических очагов или облучении малого таза (при ответе на ХT) рассматривается индивидуально. В случае небольшого размера первичной опухоли при наличии изолированного метастатического поражения поясничных лимфатических узлов возможно выполнение лимфодиссекции с последующим проведением ХЛТ расширенным полем.

\section{7. Прогрессирование РШМ}

У пациенток с рецидивом в малом тазу (без отдаленных метастазов), которым ранее проводилась ЛТ, возможны следующие варианты лечения:

- экзентерация малого таза (вопрос об экзентерации малого таза должен решаться до проведения ХT). Экстирпация матки возможна только у больных после лТ/ХЛТ по радикальной программе, если размер рецидивной опухоли в шейке матки не превышает 2 см;

- XT;

- симптоматическая терапия.

У пациенток с рецидивом в малом тазу (без отдаленных метастазов), которым ранее не проводилась ЛТ, возможны следующие варианты лечения:

- $\quad$ ХлТ

- экзентерация малого таза. 
При прогрессировании РШМ после ХЛТ следует отдать предпочтение комбинированным химиотерапевтическим режимам как более эффективным (см. раздел 3.8.).

\section{8. Химиотерапия РШМ}

Доказаны преимущества комбинированных режимов по сравнению с монохимиотерапией у пациенток с диссеминированным/рецидивирующем РШМ (табл. 6). Наибольшей эффективностью в І линии лечения обладает комбинация с использованием препаратов платины, паклитаксела и бевацизумаба. Рекомендуется проведение 6 циклов либо лечение до прогрессирования или непереносимой токсичности. С неоадъювантной целью рекомендуется проводить 2-3 курса ХТ. При прогрессировании заболевания рекомендовано определение PD-L1 и MSI в опухолевом материале. При экспрессии PD-L1 (CPS $\geq 1)$ или MSI-H/dMMRв опухоли препаратом выбора является пембролизумаб.

Таблица 6. Рекомендуемые режимы химиотерапии рака шейки матки

\begin{tabular}{|l} 
Химиолучевая терапия \\
\hline - Ц Цисплатин 40 мг/м² в/в еженедельно на фоне дистанционной лт, 6 введений
\end{tabular}

Неоадъювантная химиотерапия

- Паклитаксел 175 мг/м² в/в в 1-й день+цисплатин 75 мг/м² в/в в 1-йдень, каждые 3 нед.

- Паклитаксел 175 мг/м² в/в в 1-й день+ карбоплатин AUC 5-6 в/в в 1-йдень, каждые 3 нед.

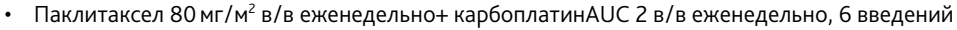

- Ифосфамид 5000 мг/м² в/в 24-часовая инфузия (+ месна 5000 мг/м²) в 1-й день+ цисплатин 75 мг/м² в/в в 1-й день, каждые 3 нед.

- Паклитаксел 175 мг/м² в/в в 1-й день+ ифосфамид 5000 мг/м² в/в 24-часовая инфузия (+ месна

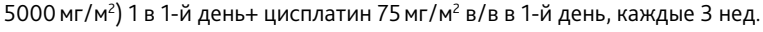

Химиотерапия I линии

- Паклитаксел 175 мг/м² в/в в 1-й день+ цисплатин 75 мг/м² в/в в 1-йдень+ бевацизумаб 15 мг/кг в 1-й день, каждые 3 нед.

- Паклитаксел 175 мг/м² в/в в 1-й день+ карбоплатин AUC 5-6 в/в в 1-й день+ бевацизума6 15 мг/кг в 1-й день, каждые 3 нед.

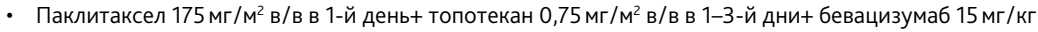
в 1-й день, каждые 3 нед.

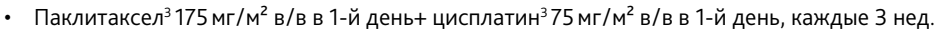

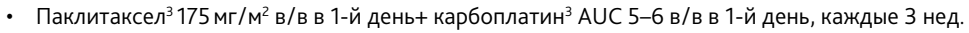

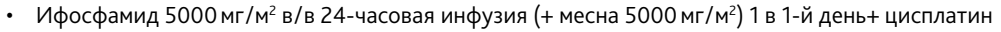
75 мг/м² в/в в 1-й день, каждые 3 нед.

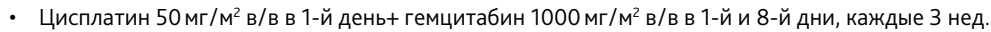

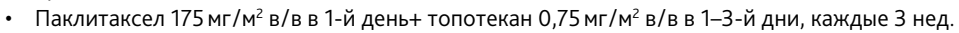

- Цисплатин 75 мг/м² в/в в 1-й день, каждые 3 нед.

- Карбоплатин AUC 5-6 в/в в 1-й день, каждые 3 нед.

Химиотерапия II линии ${ }^{4}$

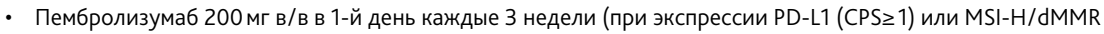
в опухоли) или пембролизумаб 400 мг в/в в 1-й день каждые 42 дня

- Митомицин 7,5 мг/м² в/в в 1-й день, каждые 6 нед.

- Иринотекан 125 мг/м² в/в в 1-й, 8-й, 15-й дни, каждые 4 нед.

- Гемцитабин 800-1000 мг/м² в/в в 1-й, 8-й, 15-й дни, каждые 3 нед. 
- Доцетаксел 75 мг/м² в/в в 1-й день, каждые 3 нед.

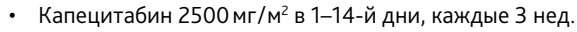

- Бевацизума6 7,5-15 мг/кг в/в в в 1-й день, каждые 3 нед.

- Топотекан 1,5 мг/м² в/в в 1-5-й дни, каждые 3-4 нед.

- Ифосфамид 1200-1500 мг/м² в/в в 1-5-й дни (+ месна 1200-1500 мг/м² в 1-5-й дни) ${ }^{1}$, каждые 3 нед.

- Винорелбин 30 мг/м² в/в в 1-й и 8-й дни, каждые 3 нед.

- Цисплатин 50 мг/м² в/в в 1-й день, каждые 3 нед. (при рецидиве в сроки> 6 мес. от окончания предшествующего лечения, включавшего производные платины)

- Карбоплатин AUC 5 в 1-й день, каждые 3 нед. (при рецидиве в сроки> 6 мес. от окончания предшествующего лечения, включавшего производные платины)

1 Месна применяется в суточной дозе, составляющей 100\% от дозы ифосфамида и разделенной на три введения в течение дня: непосредственно передвведением ифосфамида, через 4 и через 8 часов после начала его инфузии.

2 Добавление бевацизумаба кХТ рекомендуется пациенткам в удовлетворительном общем состоянии, с низким риском желудочно-кишечной и мочеполовой токсичности.

3 При наличии в анамнезе лт области малого таза начальные дозы паклитаксела, цисплатина и карбоплатина могут быть редуцированы на 20\%; при удовлетворительной переносимости возможна эскалация доз до стандартных.

${ }^{4}$ Припозднихрецидивах (>6 мес. от окончания первичного лечения) возможно повторение ХTI линии.

\section{9. Лучевая терапия РШМ}

ЛТ проводится в случаях первичного местнораспространенного опухолевого процесса по радикальной программе. Прерывание курса Лт не допускается. Продолжительность курса не должна превышать 7-8 недель. лТ может проводиться в послеоперационном периоде (адъювантно) и выполняется через 21-28 дней после операции. Оценка эффекта ЛТ/ХЛТ проводится не ранее чем через 3 мес. после окончания лечения, желательно с помощью МРТ.

\subsection{1. Дистанционная лучевая терапия}

При проведении дистанционной ЛТ предпочтительным является использование 3D-конформного облучения или лТ смодуляцией интенсивности дозы (IMRT), которая представляет собой более конформное дозовое распределение в объеме с минимальным воздействием на окружающие нормальные органы.

Дистанционная ЛТ малого таза проводится до СОД 45-50 Гр (разовая доза в России составляет 2 Гр за фракцию, в мировой практике - 1,8 Гр за фракцию 5 раз в неделю) одновременно с еженедельным введением цисплатина в дозе 40 мг/м². При противопоказаниях к введению цисплатина альтернативой является введение карбоплатина или 5-фторурацила. Возможна самостоятельная дистанционная лТ при противопоказаниях к XT.

При IMRT рекомендуется ежедневный контроль визуализации за объемом мишени, учет неопределенностей в отношении движения органов (движение матки в зависимости от степени наполнения мочевого пузыря), а также уменьшение дозовой нагрузки на органы риска. Органами риска являются мочевой пузырь, прямая, толстая и тонкая кишки, почки, головки бедренных костей. 
Расширенные поля облучения включают парааортальную область как область высокого риска прогрессирования при метастатическом поражении тазовых лимфатических узлов до уровня почечных сосудов (обычно включает L2). В случаях метастатического поражения парааортальных лимфатических узлов верхняя граница поля облучения должна находиться как минимум на уровне почечных сосудов или, по крайней мере, на 3 см выше пораженного парааортального лимфатического узла. Облучение проводится до суммарных доз 45-50 Гр одновременно с облучением малого таза. При поражении регионарных лимфатических узлов возможно увеличение суммарной дозы на эту область до 55-65 Гр в виде интегрированного буста с рекомендуемыми разовыми дозами 2,1-2,2 Гр при использовании технологии IMRT или последовательного буста при 3DCRT. Подводимые дозы зависят от размеров метастатически измененных лимфатических узлов, их локализации, вклада внутриполостной лТ и дозы за фракцию, а также доз на органы риска.

Сокращение объема ЛТ малого таза за счет исключения общих подвздошных лимфатических узлов показано при Т1b1 стадии РШМ и факторах промежуточного риска прогрессирования.

Продолжительность курса дистанционной лТ не должна превышать 5-6 нед. Однако, конформное облучение (такое как IMRT, стереотаксическая ЛТ) не заменяет брахитерапии в рутинной клинической практике лечения больных распространенных форм РШМ. Проведение ЛТ, корректируемой по изображениям (IGRT), рекомендуется как оптимальный вариант IMRT облучения, требует тщательного подхода в деталях оконтуривания всех клинических объемов, органов риска, правильной укладки пациента на столе, учета движения органов во время дыхания, возможной деформации мягких тканей вследствие распространения опухолевого процесса в малом тазу; требуется тщательное дозиметрическое планирование квалифицированным медицинским физиком. Применение IGRT обеспечивает точную воспроизводимость запланированного распределения дозы, нивелирования погрешностей, связанных с подвижностью «мишени» и окружающих нормальных тканей, что, в свою очередь, позволяет уменьшить отступы при планировании объема облучения и снизить лучевую нагрузку на органы риска.

\subsection{2. Внутриполостная лучевая терапия (брахитерапия)}

Брахитерапия является определяющим и обязательным этапом лечения, при котором заданный локальный объем облучения получает максимальную дозу воздействия практически эквивалентно равную общей дозе, достигнутой от дистанционного этапа облучения. Таким образом, проведение брахитерапии является основным прогностическим фактором в локальном контроле РШМ.

В случаях самостоятельного курса ХЛТ/ЛТ местнораспространенного РШМ рекомендуется повторная МРТ с в/в контрастированием после дистанционной ЛТ для оценки степени резорбции первичного объема опухоли перед проведением брахитерапии. Брахитерапия является малоинвазивным хирургическим методом лечения. Проводится с использованием общей седации или соответствующей анестезии. В зависимости от степени инвазивности процедуры выбирают объем анестезиологического пособия для достижения максималь- 
ного эффекта от лечения и комфортного состояния больной - общая (внутривенная), регионарная (эпидуральная), местная.

Рекомендуется проводить брахитерапию под визуальным контролем, с использованием МРТ или КТ изображений для планирования дозового распределения. Альтернативно методом визуального контроля может быть УЗИ. Брахитерапия проводится в конце 4-5-й недели (при малых объемах опухоли до 4 см) или после ХлТ (при больших объемах опухоли более 4 см).

При брахитерапии учитываются объемы для мишени: резидуальный (остаточный) объем опухоли шейки матки GTV-Tres (после этапа ХЛТ/лT), адаптивный клинический объем опухоли высокого риска CTV-THR (включает всю шейку матки и остаточную опухолевую инфильтрацию), а также клинический объем промежуточного риска CTV-TIR.

При выявлении на момент проведения брахитерапии остаточной опухолевой инфильтрации в параметрии, а также распространения опухоли за пределы шейки матки, например, на влагалище, тело матки, или прилегающий орган, эти мишени включаются в объем CTV-HR.

Используются стандартные аппликаторы для внутриполостной брахитерапии - центральный маточный интрастат и овоиды/кольцевой влагалищный аппликатор.

В случаях распространенного опухолевого процесса шейки матки на момент брахитерапии используются иглы-интрастаты дополнительно к стандартному аппликатору для оптимизации дозового распределения на объем опухоли (гибридная внутриполостная/внутритканевая брахитерапия) и реализации предписанной дозы на органы риска.

Суммарные дозы брахитерапии составляют 40-45 Гр (EQD2), таким образом, суммарные дозы полного курса лТ, включающего дистанционную ЛТ и брахитерапию, составляют на CTV-HR $\geq 80$ Гр (EQD90), CTV-IR $\geq 60$ Гр (D98), GTV-Tres $\geq 90$ Гр (D98).

При отсутствии технической возможности использования технологии IMRT с визуальным контролем возможно проведение самостоятельной 3D-конформной дистанционной ЛТ или ХЛТ (с применением цисплатина) $о$ облучение парааортальной области в сочетании c 2D 6рахитерапией. Следует придерживаться тех же принципов выделения клинических объемов, фракционирования, продолжительности курса ЛТ. Для локального буста на область лимфатических тазовых узлов рекомендуется применять последовательный буст после окончания курса 3D-конформной лт.

Для 2D брахитерапии контроль расчета дозы остается по точке A. Суммарная доза в точке А должна быть $\geq 75$ Гр (EQD2) при малых объемах CTV-THR ( $\leq 3$ см) и выше - при CTV-THR (>4 cm). В отчетности необходимо отмечать максимальный размер CTV-THR. Рекомендуемые точки ограничения для 2D брахитерапии и объемы 3D для прямой кишки, мочевого пузыря, влагалища, сигмовидной кишки должны основываться на опубликованных клинических рекомендациях.

Суммарные минимальные и максимальные дозы на органы риска составляют:

- D2cc (минимальная доза в 2-х см³) на мочевой пузырь - <80-90 Гр (EQD2 $\left.{ }_{3}\right)$

- $\quad$ D2cc (минимальная доза в 2-х см³) на прямую кишку - <65-75 Гр (EQD2 $\left.{ }_{3}\right)$

- D2cc (минимальная доза в 2-х см ${ }^{3}$ ) на сигмовидную кишку - <70-75 Гр (EQD2 $\left.{ }_{3}\right)$

- D2cc (минимальная доза в 2-х см³) на кишечник - <70-75 Гр (EQD2 $)$ 
- D2cc (минимальная доза в 2-х см³) на влагалище (ректо-вагинальная область) $<65-70$ Гр $\left(\mathrm{EQD2}_{3}\right)$.

Точка А должна являться отправной точкой для оптимизации плана облучения, хотя точка А трансформировалась в отчетность значений распределения дозы по объемам. Курс брахитерапии проводится за 3-4 фракции. Дистанционная ЛТ с целью подведения локальных бустов на шейку матки, параметрии по объемам GTV-Tres, CTV-THR, CTV-TIR не рекомендуется в рутинной практике даже в случаяхиспользования стереотаксических технологий. Применение центрального блока для параметрального буста при дистанционной конформной лТ не рекомендуется.

\subsection{3. Адьювантная лучевая или химиолучевая терапия}

Адьювантная ЛТ или ХЛТ проводится по аналогичным принципам планирования объемов облучения и фракционирования, применяющимся в самостоятельных программах. Адьювантная (дополнительная) брахитерапия в послеоперационном периоде рекомендуется при высоком риске прогрессирования - положительном крае резекции слизистой влагалища. В случаях более глубокого поражения края резекции (например, параметрально или паравагинально) или макроскопически положительном крае границ необходимо использовать сложные конструкции аппликаторов (например, многоканальный влагалищный аппликатор) или иглы-интрастаты для внутритканевой брахитерапии сцелью подведения максимально адекватной дозы облучения. Для областей повышенного риска, где невозможно провести брахитерапию, рассматривается возможность увеличения дозы облучения с использованием дистанционной лт.

\section{4. РАК ШЕЙКИ МАТКИ И БЕРЕМЕННОСТЬ}

Пациенткам, не желающим сохранять беременность, выполняют прерывание беременности и проводят лечение в соответствии со стандартами лечения дисплазий и РШМ.

При выявлении РШМ во время желанной беременности необходимо направлять пациенток в специализированные центры с возможностью ведения и лечения данной категории больных мультидисциплинарной командой онкологов, акушеров-гинекологов, неонатологов и возможностью интенсивной помощи недоношенным новорожденным. При диагностике РШМ во время беременности рекомендовано клиническое обследование, гистологическая верификация диагноза, выполнение УзИ и/или МРТ. Рентгенологические исследования и КТ во время беременности возможны только при экранировании матки

При подозрении на метастатическое поражение лимфатических узлов по данным объективных методов обследования необходима морфологическая верификация ихметастатического поражения.

Тактика лечения определяется в зависимости от стадии заболевания и срока беременности.

- При выявлении LSIL по данным кольпоскопии и цитологического исследования мазков с шейки матки беременность пролонгируют. Цитологическое исследование ш/матки 
выполняют в каждом триместре. Через 6-8 недель после родов выполняют повторное обследование - кольпоскопия, цитологическое исследование мазков с шейки матки.

- При выявлении HSIL по данным кольпоскопии и цитологического исследования мазков с шейки матки, под кольпоскопическим контролем выполняется прицельная биопсия наиболее измененных участков шейки матки с помощью биопсийных щипцов. Получают 2-5 биоптатов объемом 1-5 мм³. Если при кольпоскопии выявляются признаки инвазивного рака (атипичные сосуды), то показана эксцизионная петлевая биопсия (рекомендуемая глубина иссечения 6-10мм). При гистологическом подтверждении диагноза CIN II-III беременность пролонгируют, кольпоскопию и цитологическое исследование мазков с шейки матки проводят в каждом триместре беременности. Через 6-8 недель после родов выполняют повторное обследование, при подтверждении диагноза CIN III показана конизация шейки матки, выскабливание оставшейся части цервикального канала. При отсутствии подтверждения HSIL после беременности проводится тщательный цитологический мониторинг.

- Единственным показанием к конизации (широкой петлевой эксцизии) шейки матки во время беременности является микроинвазивный рак шейки матки по данным биопсии. Конизация (широкая петлевая эксцизия) шейки матки позволяет разграничить больных микроинвазивным раком, которых можно наблюдать, и больных, которым показано дальнейшее лечение.

- При РШМ IA1 стадии в отсутствие опухолевых клеток по краю резекции больную можно наблюдать до завершения беременности. Родоразрешение путем кесарева сечения в этом случае следует проводить только по акушерским показаниям.

- При выявлении опухолевых эмболов в лимфатических щелях у больных РШМ ІА1 стадии по результатам гистологического исследования конуса шейки матки в отсутствие опухолевых клеток по краю резекции больной во II триместре беременности показана двусторонняя тазовая лимфодиссекция, которая может выполняться внебрюшинным доступом открыто или эндоскопически. Рекомендуется выполнение лимфодиссекции до 22 недели беременности. При отсутствии метастазов в тазовых лимфатических узлах беременность пролонгируют. Родоразрешение путем кесарева сечения в этом случае следует проводить только по акушерским показаниям.

- У пациенток IA2 стадии (установленной по результатам конизации) во II триместре беременности целесообразна двусторонняя тазовая лимфодиссекция, которая может выполняться внебрюшинным доступом открыто или эндоскопически. Рекомендуется выполнение лимфодиссекции до 22 недели беременности. При отсутствии метастазов в тазовых лимфатических узлах беременность пролонгируют до 37-38 недели. Родоразрешение путем кесарева сечения с одномоментным выполнением модифицированной расширенной экстирпации матки (тип II/B). При желании пациентки сохранить детородную функцию трахелэктомию (тип II/B) выполняют отсрочено, через 4-6 недель.

- При выявлении метастазов в тазовых лимфатических узлаху пациенток IA1 или IA2 стадий РШМ во II триместре беременности целесобразно либо прерывание беременности и лечение в соответствии со стадией заболевания либо, при настоятельном желании женщины пролонгировать беременность, возможно проведение неоадъювантной XT. 
- При выявлении IA1 или IA2 стадий РШМ (по данным конизации) в III триместре беременность пролонгируют до 37-38 недели. Родоразрешение путем кесарева сечения. Через 4-6 недель после родов выполняется конизация шейки матки и выскабливание оставшейся части цервикального канала. Далее тактика лечения определяется по результатам гистологического исследования удаленного конуса.

- При установлении диагноза IB1 стадии РШМ в I триместре беременности лечение, как правило, начинают сразу. Однако если женщина настаивает на сохранении беременности, возможно пролонгировать ее до II триместра, когда выполняется тазовая лимфодиссекция (до 22 недели беременности). При выявлении метастазов в тазовых лимфатических узлах рекомендовано проведение неоадъювантной ХT.

- При отсутствии метастазов в тазовых лимфатических узлах у больных РШМ IB1 стадии с опухолями менее 2 см во II триместре беременности возможно отложить лечение до момента родоразрешения или выполнить простую трахелэктомию (тип I/A). Однако необходимо помнить, что проведение органосохраняющих операций на фоне беременности связано с высоким риском прерывания последней.

- При отсутствии метастазов в тазовых лимфатических узлах у больных РШМ IB1 стадии с опухолями 2-4 см во II триместре беременности, рекомендовано проведение неоадъювантной XT, которую следует закончить за 3 недели до планируемой даты родоразрешения.

- При установлении диагноза РШМ IB1 стадии после 22 недели беременности возможно проведение неоадъювантной ХТ или отсрочка начала лечения до момента родоразрешения.

- У больных РШМ $\geq$ ІВ2 стадии при сроках беременности до 22 недель единственным возможным способом сохранения беременности является проведение неоадъювантной ХТ с начала II триместра (не ранее 14 недели беременности).

- При установлении диагноза РШМ $\geq \mathrm{IB2}$ стадии после 22 недели беременности возможно проведение неоадъювантной ХТ или отсрочка начала лечение до момента родоразрешения.

- В качестве неоадъювантной ХТ рекомендуется применять режимы на основе препаратов платины. Наиболее часто применяется режим цисплатин 75 мг/м² или карбоплатинAUC 5 и паклитаксел 175 мг/м² с интервалом 3 недели. ХT следует закончить за 3 недели до планируемой даты родоразрешения. Родоразрешение выполняется путем кесарева сечения. После родоразрешения лечение проводится в соответствии со стадией заболевания с учетом проведенного ранее лечения (неоадъювантная ХT).

\section{5. ДИСПАНСЕРНОЕ НАБЛЮДЕНИЕ}

- Физикальное обследование, в том числе гинекологический осмотр - каждые 3 мес. в течение первых 2 лет, каждые 6 мес.-в течение 3-го и 4-го года, затем-ежегодно.

- Ц Цитологическое исследование мазков со слизистой культи влагалища - каждые 3 мес. в течение первых 2 лет, каждые 6 мес.-в течение 3-го и 4-го года, затем-ежегодно. 
В случае подозрения на рецидив рекомендована биопсия с гистологическим исследованием.

- УзИ брюшной полости, малого таза и забрюшинного пространства и определение уровня SCC (при плоскоклеточном раке) - каждые 3 мес. в течение первых 2 лет, каждые 6 мес.- в течение 3-го и 4-го года, затем - ежегодно.

- R-графия органов грудной клетки - ежегодно.

- KT/MPТ - по показаниям. 